\title{
Magnetic Phase Diagram of Layered Manganites in the Highly Doped Regime
}

\author{
J.F. Mitchell, C.D. Ling, J.E. Millburn, D.N. Argyriou, A. Berger, M. Medarde \\ Materials Science Division \\ Argonne National Laboratory, Argonne, IL 60439
}

\begin{abstract}
The submitted manuscript has been created by the University of Chicago as Operator of Argonne National Laboratory ("Argonne") under Contract No. W-31-109-ENG-38 with the U.S. Department of Energy. The U.S. Government retains for itself, and others acting on its behalf, a paid-up, nonexclusive, irrevocable worldwide license in said article to reproduce, prepare derivative works, distribute copies to the public, and perform publicly and display publicly, by or on behalf of the Government.
\end{abstract}

Submitted for the Proceedings of the 8th Joint Magnetism and Magnetic Materials-INTERMAG Conference, San Antonio, TX, Jan. 7-11, 2001, to be published by IEEE Trans. Mag.

Work supported by the U.S. Department of Energy, Office of Science under contract \#W-31-109-ENG-38. 


\section{DISCLAIMER}

This report was prepared as an account of work sponsored by an agency of the United States Government. Neither the United States Government nor any agency thereof, nor any of their employees, make any warranty, express or implied, or assumes any legal liability or responsibility for the accuracy, completeness, or usefulness of any information, apparatus, product, or process disclosed, or represents that its use would not infringe privately owned rights. Reference herein to any specific commercial product, process, or service by trade name, trademark, manufacturer, or otherwise does not necessarily constitute or imply its endorsement, recommendation, or favoring by the United States Government or any agency thereof. The views and opinions of authors expressed herein do not necessarily state or reflect those of the United States Government or any agency thereof. 


\section{DISCLAIMER}

Portions of this document may be illegible in electronic image products. Images are produced from the best available original document. 


\section{MAGNETIC PHASE DIAGRAM OF LAYERED MANGANITES IN THE HIGHLY DOPED REGIME}

RECEIVED DEC 0 \& 2000

OSTI

Materials Science Division, Argonne National Laboratory, 9700 S. Cass Ave., Argonne, IL 60439

\section{Abstract}

The naturally layered colossal magnetoresistive (CMR) manganites $\mathrm{La}_{2-2 x} \mathrm{Sr}_{1+2 x} \mathrm{Mn}_{2} \mathrm{O}_{7}$ exhibit an extremely varied range of magnetic and electronic behavior over a very narrow composition range between $x=0.3$ and $x=0.5$. The successful synthesis in our laboratories of compounds with nominally greater than 50 percent $\mathrm{Mn}^{4+}$ concentration has now allowed the study of this heretofore unexplored region of the phase diagram. Here we present detailed neutron diffraction measurements of these compounds with doping levels $0.5<\mathrm{x}<1.0$. As predicted by simple theories, the type-A layered antiferromagnetic (AF) structure is found at $x \sim 0.5$ and the type-G "rocksalt" AF structure at $x=1.0$. Between these two extremes is found a C-type structure (ferromagnetic rods parallel to $b$ coupled antiferromagnetically to all neighboring rods) stabilized by orbital ordering of $y^{2}$ states. Also in this $\mathrm{Mn}^{4+}$-rich regime is found a region in which no long-range magnetic order is observed. We discuss how semi-empirical models can explain the variety of magnetic structures and how structural trends as a function of doping corroborate the unifying notion of a shift from in-plane to axial orbital occupation as the $\mathrm{Mn}^{4+}$ concentration is decreased. 


\section{Introduction}

Mixed-valent manganese oxide materials exhibit an astonishing array of collective phenomena that depend on a delicate balance among magnetic, electronic, and lattice degrees of freedom. Recent years have witnessed a wide-ranging interest in the study of these transition metal oxides, focussing on 3-D perovskites, $\mathrm{A}_{1-\mathrm{x}} \mathrm{B}_{\mathrm{x}} \mathrm{MnO}_{3}(\mathrm{~A}=\mathrm{rare}$ earth, $B=$ alkaline earth).[1-3] A driving force behind such studies has been an attempt to understand-and perhaps harness for applications-the phenomenon of colossal magnetoresistance (CMR) characteristic of these perovskites. Another class of CMR materials related to the perovskites is the so-called Ruddlesden-Popper phase $\mathrm{La}_{2}$. ${ }_{2 x} \mathrm{Sr}_{1+2 x} \mathrm{Mn}_{2} \mathrm{O}_{7}$, (Fig. 1, inset) first prepared by MacChesney, et al.[4] in powder form and more recently in single-crystal form by Kimura, et al.[5] Such layered materials offer the opportunity to explore the interplay among spin, charge, and lattice under conditions of reduced dimensionality, where magnetic and/or electronic fluctuations should be enhanced relative to the 3-D analogs. A number of groups have addressed this point in the doping region $0.3<\mathrm{x} \leq 0.5$, where ferromagnetic ground states and insulator-metal transitions are found.[6-9] Unfortunately, because of the difficulty in preparing singlephase samples outside this narrow doping range, little is known about the broad trends in structural and magnetic properties of this series.

In this work we present the successful preparation of highly-doped layered manganites $\mathrm{La}_{2-2 x} \mathrm{Sr}_{1+2 x} \mathrm{Mn}_{2} \mathrm{O}_{7}(0.5 \leq \mathrm{x} \leq 1.0)$ in ceramic form and report the determination of the full crystallographic and magnetic phase diagrams of these $\mathrm{Mn}^{4+}$-rich compounds. Using these phase diagrams, we show that Goodenough's semi-empirical models for MnO-Mn magnetic exchange in perovskites are equally applicable to the 2-D layered 
compounds. Importantly, a sequence of magnetic structures $v s \mathrm{Mn}^{4+}$ content very similar to the 3-D perovskites is obtained. Building on these observations, we provide (a) direct evidence of orbital ordering and how it impacts spin-ordering and (b) indirect evidence for a shift of orbital occupation from in-plane to axially directed $e_{\mathrm{g}}$ states as the $\mathrm{Mn}^{4+}$ concentration is decreased, in accordance with recent theoretical treatments of this system.[10,11]

\section{Experimental}

Samples of $\mathrm{La}_{2-2 \mathrm{x}} \mathrm{Sr}_{1+2 \mathrm{x}} \mathrm{Mn}_{2} \mathrm{O}_{7.8}$ were synthesized by combining stoichiometric quantities of high purity $\mathrm{La}_{2} \mathrm{O}_{3}$ (prefired at $1000{ }^{\circ} \mathrm{C}$ ), $\mathrm{SrCO}_{3}$, and $\mathrm{MnO}_{2}$. The mixtures were calcined at $900^{\circ} \mathrm{C}$ then prefired at $1200^{\circ} \mathrm{C}$ to yield multiphase precursor materials. Single-phase samples were obtained as dark brown $(\mathrm{x} \sim 1.0)$ to black $(\mathrm{x} \sim 0.5)$ pellets by firing the precursor materials at $1650^{\circ} \mathrm{C}$ in air for $18 \mathrm{hrs}$, followed by rapid quenching into dry ice. In this way the entire range $0.5 \leq \mathrm{x} \leq 1.0$ was prepared at intervals $\Delta x=0.02$. Samples thus synthesized form with substantial oxygen-deficiency $(\delta>0.0)$.[12] Oxygen-stoichiometric samples (i.e., $\delta=0.0$ ) were obtained by annealing the as-prepared samples in $100 \% \mathrm{O}_{2}$ at $425{ }^{\circ} \mathrm{C}$ for $12 \mathrm{hrs}$. All oxygen vacancy concentrations were measured both by thermogravimetric analysis (TGA) and by iodometric titration, and in certain cases corroborated by full-profile refinement of neutron powder diffraction data.

Temperature-dependant time-of-flight (TOF) neutron powder diffraction data were collected on the Special Environment Powder Diffractometer (SEPD), the High Intensity Powder Diffractometer (HIPD) and the General Purpose Powder Diffractometer (GPPD) at Argonne National Laboratory's Intense Pulsed Neutron Source (IPNS). Data 
were analysed using the program suite GSAS[13]. The perovskite $\mathrm{La}_{1-x} \mathrm{Sr}_{x} \mathrm{MnO}_{3}$ was included in a number of refinements as a minor impurity, $0-3 \mathrm{wt} \%$. The difference between the neutron scattering lengths of $\mathrm{La}$ and $\mathrm{Sr}$ was insufficient to adequately assess possible ordering on mixed $\mathrm{La} / \mathrm{Sr}$ sites, therefore these sites were treated as disordered mixtures of $\mathrm{La}$ and $\mathrm{Sr}$ in ratios appropriate to the value of $\mathrm{x}$. Oxygen sites for annealed samples were fixed at $100 \%$ occupancy in accordance with thermogravimetric analyses. Unit cells, atomic positions, isotropic thermal displacement parameters, absorption and extinction of the samples were refined in addition to the background and peak profiles of the diffraction patterns.

\section{Results and Discussion}

The successful synthesis of the layered manganites $\mathrm{La}_{2-2 x} \mathrm{Sr}_{1+2 x} \mathrm{Mn}_{2} \mathrm{O}_{7}(0.5 \leq \mathrm{x} \leq$ 1.0) requires a two-step method, highlighting that these phases are metastable under the normal conditions under which manganites are prepared $\left(\mathrm{T} \sim 1300^{\circ} \mathrm{C}\right.$, air). Furthermore, the crystal chemistry of these metastable phases suggests a possible avenue to modify the magnetic and/or electronic structure of the active bilayer unit. By quenching from extremely high temperature $\left(T=1650^{\circ} \mathrm{C}\right)$, where the $n=2 R-P$ structure apparently is in equilibrium, single phase metastable materials can be isolated. Not unexpectedly, such high temperature conditions are favor the reduction of $\mathrm{Mn}$ to an average oxidation state below the nominal value of $3+\mathrm{x}$. As we have reported earlier, this reduction is accommodated by the introduction of oxygen vacancies into the crystal structure.[12] In an earlier report on the $\mathrm{Sr}_{3} \mathrm{Mn}_{2} \mathrm{O}_{7-\delta}(\mathrm{x}=1.0)$ end-member,[14] we pointed out that the oxygen vacancies lie in the $\mathrm{MnO}_{2}$ planes, an unusual location for such vacancies, which 
typically locate between the $\mathrm{MnO}_{2}$ sheets in related compounds.[15] Refinement of room temperature neutron powder data on as-made samples with varying $\mathrm{La} / \mathrm{Sr}$ ratio reveals that the oxygen vacancies decrease in number and migrate into this more commonly found axial site with increasing La content (Table I). We have previously shown that the variation in oxygen vacancy concentration reflects a tendency of the system to establish an invariant average $\mathrm{Mn}$ oxidation state fixed by synthesis conditions.[12] By appropriate tuning of the oxygen vacancy concentration in the axial site, it may be possible to electronically and/or magnetically decouple the $\mathrm{MnO}_{2}$ layers, effectively reducing the dimensionality of the system.[16] Experiments to test this hypothesis are currently underway.

Treating the oxygen-deficient materials $\mathrm{La}_{2-2 x} \mathrm{Sr}_{1+2 x} \mathrm{Mn}_{2} \mathrm{O}_{7-\delta}$ at $450{ }^{\circ} \mathrm{C}$ in oxygen rapidly ( $<4$ hrs) fills all of the vacancies, yielding stoichiometric materials (i.e., $\delta=0.0$ ) as confirmed by neutron powder diffraction. These stoichiometric materials have been used to simultaneously determine the crystallographic and magnetic phase diagram via temperature-dependent neutron powder diffraction. Fig. 1 shows the results of these experiments and reveals the rich variety of phases exhibited by these $\mathrm{Mn}^{4+}$-rich compounds.

Concentrating initially on the magnetic phase diagram, we find considerable similarity to the perovskite phase diagram rationalized by Goodenough[17] in terms of the overlap of occupied and unoccupied $\mathrm{e}_{\mathrm{g}}$ orbitals on the Mn sites. In this simple but powerful picture, ferromagnetic exchange arises from the overlap of occupied and unoccupied $d$ orbitals in the Mn-O-Mn unit, requiring that at least one member of the pair be $\mathrm{Mn}^{3+}$. All $\mathrm{Mn}^{4+}-\mathrm{Mn}^{4+}$ interactions are antiferromagnetic, as are interactions 
involving $\mathrm{Mn}^{3+}$ in which the occupied $\mathrm{e}_{\mathrm{g}}$ orbital is directed orthogonal to the Mn-O-Mn axis. In the present system, we see a progression of magnetic structures with increasing Sr concentration (equivalently, increasing $\mathrm{Mn}^{4+}$ concentration) from A-type (ferromagnetic sheets coupled antiferromagnetically) through C-type (ferromagnetic rods coupled antiferromagnetically) to G-type (all five $\mathrm{Mn}-\mathrm{Mn}$ neighbors antiferromagnetically coupled). Thus, in accordance with Goodenough's picture, the increasing concentration of $\mathrm{Mn}^{4+}$ in the lattice leads to progressively less ferromagnetism (sheets $\rightarrow$ rods $\rightarrow$ points) as the number of unoccupied $\mathrm{e}_{\mathrm{g}}$ orbitals increases. An unusual region exists for $\mathrm{Sr}$ concentrations $0.66<\mathrm{x}<0.72$ in which no long-range magnetic order is detected by neutron diffraction for $\mathrm{T}>5 \mathrm{~K}$. The nature of this unexpected region is currently being investigated with the speculation that regions of the A-type and C-type phases coexist on a length scale shorter than that probed by diffraction techniques.

As shown in Fig. 1, samples taken from the region $0.74<x<0.90$ adopt the Ctype (ferromagnetic rods) magnetic structure.[18] In addition, a crystallographic phase transition from tetragonal $(\mathrm{I} / \mathrm{mmm})$ to orthorhombic $(\mathrm{Immm})$ symmetry is observed at temperatures approximately $100 \mathrm{~K}$ above the Neél temperature throughout this region. Detailed study shows that the structural phase transition is consistent with an orbital ordering that "locks-in" the material for the specific C-type magnetic structure. First, consider the orthorhombic structure. The rather substantial splitting of the $a$-and $b$-axis lengths (see Fig. 3) indicates that a predominance of electron density is being added to orbitals directed along $b$. In fact, as shown in Fig. 3 , the $a$-axis exhibits virtually no concentration dependence in the orthorhombic regime $(T=20 \mathrm{~K})$. These observations are consistent with doped electrons entering $y^{2}$ orbitals to precipitate a strain-driven 
structural phase transition. Within the Goodenough framework, the interaction of these directed orbitals with unoccupied $\mathrm{Mn}^{4+}$ sites neighboring along the $b$-axis should yield ferromagnetic rods at sufficiently low temperature. Because the orbitals are lined up preferentially along the $b$-axis, occupied orbitals on $\mathrm{Mn}$ sites from adjacent rods will be orthogonal, yielding the antiferromagnetic arrangement characteristic of the C-type structure. A schematic of this ordering process is shown in Fig. 2, which illustrates how the specific orbital ordering prepares the system to adopt its unique magnetic ordering scheme.

In the case of the perovskite system described by Goodenough, the A-type structure is not found in the region $x \sim 0.5$. However, the particular crystal structure of the layered material offers an opportunity to explain this discrepancy within the general framework of Goodenough's magnetic exchange rules. Recent theoretical treatments by Akimoto[10] and independently by Maezono.[11] have proposed that the occupied orbital state for $\mathrm{x} \sim 0.5$ is an $\mathrm{x}^{2}-\mathrm{y}^{2}$ planar orbital, while for $\mathrm{x}<0.5$ the axial $\mathrm{z}^{2}$ orbital is predominantly occupied.[19] A plot of the $a$-and $c$-axis lattice constants at room temperature and at $20 \mathrm{~K}$ as a function of Sr content is shown in Fig. 3. Upon doping La into the $\mathrm{x}=1.0$ end-member the $a$-axis expands and the $c$-axis contracts. Below $\mathrm{x} \sim 0.5$, however, this trend reverses, and the $c$-axis dramatically expands while the $a$-axis levels off, dropping slightly as $\mathrm{x}$ approaches 0.3 . These trends are consistent with the addition of electron density in the $\mathrm{MnO}_{2}$ planes for $0.5<\mathrm{x} \leq 1.0$ followed by a shift into axial occupation for $\mathrm{x}<0.5$, in accordance with the theoretical predictions of Akimoto and Maezono. 
Unfortunately, our data cannot identify the symmetry of the occupied in-plane orbital in the A-type stability field. However, the $x^{2}-y^{2}$ orbital should give rise to ferromagnetic sheets, as all interactions between $\mathrm{Mn}^{3+}$ and $\mathrm{Mn}^{4+}$ sites within the plane will be ferromagnetic; the occupied orbital extends isotropically toward all four neighboring sites. Furthermore, these ferromagnetic sheets should be coupled antiferromagnetically to yield the A-type structure because pairs of $x^{2}-y^{2}$ orbitals in adjacent planes have zero overlap. The crystal structure data thus provide an indirect signature of orbital ordering consistent with the A-type spin ordering. Finally, since we know that for $0.8<\mathrm{x} \leq 1.0$ anisotropic $\mathrm{y}^{2}$ orbitals are occupied (vide supra), it is important to identify and to explore how the system crosses over to occupation of $x^{2}-y^{2}$ orbitals, perhaps using recently developed tools in resonant $\mathrm{x}$-ray scattering[20].

\section{Conclusions}

The highly-doped layered manganites provide an exceptional opportunity to explore the relationship between crystal structure and magnetic exchange. The successful synthesis of the series $0.5 \leq \mathrm{x} \leq 1.0$ has allowed the complete determination of this relationship via the structural and magnetic phase diagrams. Significantly, we found that orbital ordering patterns inferred from structural trends consistently explain the magnetic structure within the simple Goodenough framework. The existence of a "gap" region with no long-range order and the possibility of tuning the magnetic/electronic states via control of oxygen vacancies demonstrate that layered manganites hold much promise for future exploration of the connections among spin, charge, and lattice degrees of freedom in transition metal oxides. 


\section{Acknowledgements}

The authors wish to thank Simine Short for invaluable assistance in the collection of neutron powder data on the SEPD and Heloisa Bordallo for data collection and interpretation on the HIPD instrument. This work was supported by the U.S. Department of Energy Office of Science under Contract No. W-31-109-ENG-38. 


\section{References}

1. A. Urushibara, et al. Phys. Rev. B 51, 14103 (1995)

2. P. Schiffer, et al. Phys Rev. Lett. 75, 3336 (1995)

3. D.E. Cox, P.G. et al. Phys. Rev. B 57, 3305 (1998)

4. J.B. MacChesney, et al. J. Appl. Phys. 40, 1243 (1969)

5. T. Kimura, et al. Science 274,1698 (1996)

6. K. Hirota, et al. J. Phys. Soc. Jpn. 67, 3380 (1998).

7. J. Q. Li, et al. Phys. Rev. B 57, 3205R (1998).

8. P. D. Battle, et al. Chem. Mater. 9, 1042 (1997).

9. L. Vasiliu-Doloc, et al. Phys. Rev. Lett, 83, 4393 (1999).

10. T. Akimoto, et al. Phys. Rev. B 59, R14153 (1999).

11. R. Maezono and N. Nagaosa, Phys. Rev. B 61, 1825 (2000).

12. J. E. Millburn, et al. Chem. Comm. 15, 1389 (1999).

13. A. C. Larson and R. B. V. Dreele,, (Los Alamos National Laboratory, 1990).

14. J. F. Mitchell, et al. J. Solid State Chem. 141, 599 (1998).

15. S. E. Dann and M. T. Weller, J. Solid. State Chem. 115, 499 (1995).

16. The $\mathrm{n}=1$ member of the R-P series, $\mathrm{La}_{1-\mathrm{x}} \mathrm{Sr}_{1+\mathrm{x}} \mathrm{MnO}_{4}$ shows no ferromagnetic ground states or insulator metal transitions implicating the importance of the bilayer unit.

17. J. B. Goodenough, Phys. Rev. 100, 564 (1955).

18. In fact, in this region we find magnetic polytypism with two C-type phases: one with the crystallographic $c$-axis and one with a doubled $c$-axis. It is likely that 
this polytypism arises because of the weak magnetic coupling between the bilayers.

19. Photoemission measurements (e.g., D. Dessau, et al. Phys. Rev. Lett. 81, 192 (1998)) on $\mathrm{x}=0.4$ samples indicate that these orbitals are nearly degenerate, implying that a shift of electron density between the orbital manifolds may not be energetically costly.

20. Y. Murakami, et al. Phys. Rev. Lett. 80, 1932 (1998) 
Table I. Refined room temperature oxygen site occupancies in $\mathrm{La}_{2-2 x} \mathrm{Sr}_{1+2 x} \mathrm{Mn}_{2} \mathrm{O}_{7-\delta}$

\begin{tabular}{lllll} 
& \multicolumn{4}{c}{$\mathrm{x}$ in $\mathrm{La}_{2-2 x} \mathrm{Sr}_{\mathrm{I}+2 \times} \mathrm{Mn}_{2} \mathrm{O}_{7-\delta}$} \\
\cline { 2 - 5 } & 0.60 & 0.75 & 0.85 & 1.0 \\
\hline$\delta$ & $0.114(9)^{\mathrm{a}}$ & $0.263(13)$ & $0.355(21)$ & $0.507(1)$ \\
In-plane & $1.0^{\mathrm{b}}$ & $0.958(5)$ & $0.921(8)$ & $0.875(5)$ \\
Axial & $0.886(9)$ & $0.905(8)$ & $0.961(14)$ & $0.993(11)$ \\
\hline
\end{tabular}

${ }^{2}$ Numbers in parentheses are estimated standard deviations. ${ }^{6}$ Fixed during refinement. 


\section{Figure Captions}

Figure 1. Crystallographic and magnetic phase diagram of $\mathrm{La}_{2,2 x} \mathrm{Sr}_{1+2 x} \mathrm{Mn}_{2} \mathrm{O}_{7}$ determined by neutron powder diffraction. Inset: crystal structure of the $\mathrm{n}=2 \mathrm{R}-\mathrm{P}$ phase. $\mathrm{FM}=$ ferromagnetic metal $\mathrm{CAF}=$ canted antiferromagnet (not discussed in text)

Figure 2. Orbital ordering and magnetic transition in phases from the region $x \sim 0.75$.

Figure 3. Variation of lattice constants with $\mathrm{Sr}$ content, $\mathrm{x}$, at room temperature (solid line) and at $20 \mathrm{~K}$ (dashed line) 


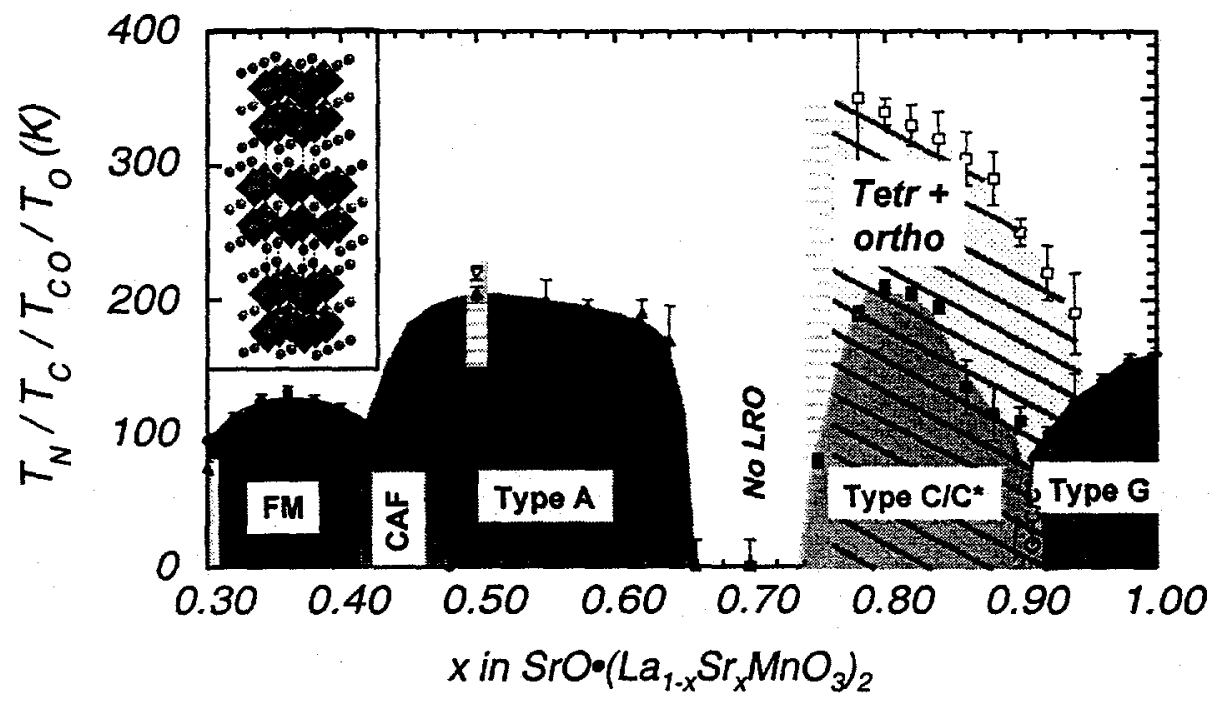

Figure 1. -- Mitchell, et al. "Magnetic Phase Diagram ..." 

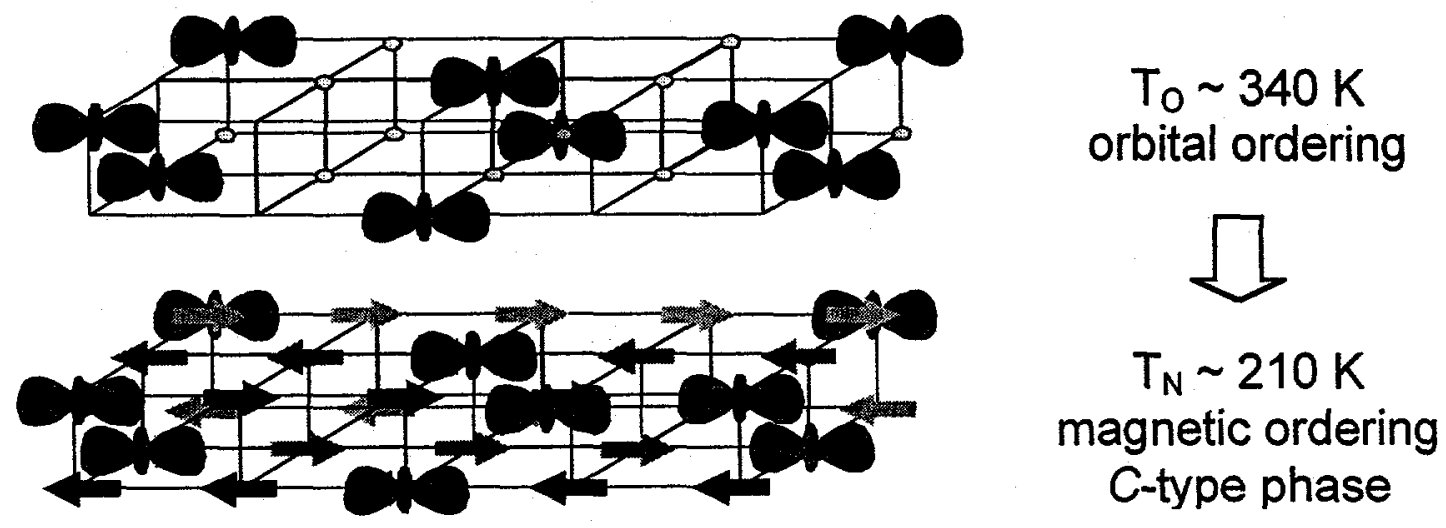

Figure 2. -- Mitchell, et al. "Magnetic Phase Diagram ..." 


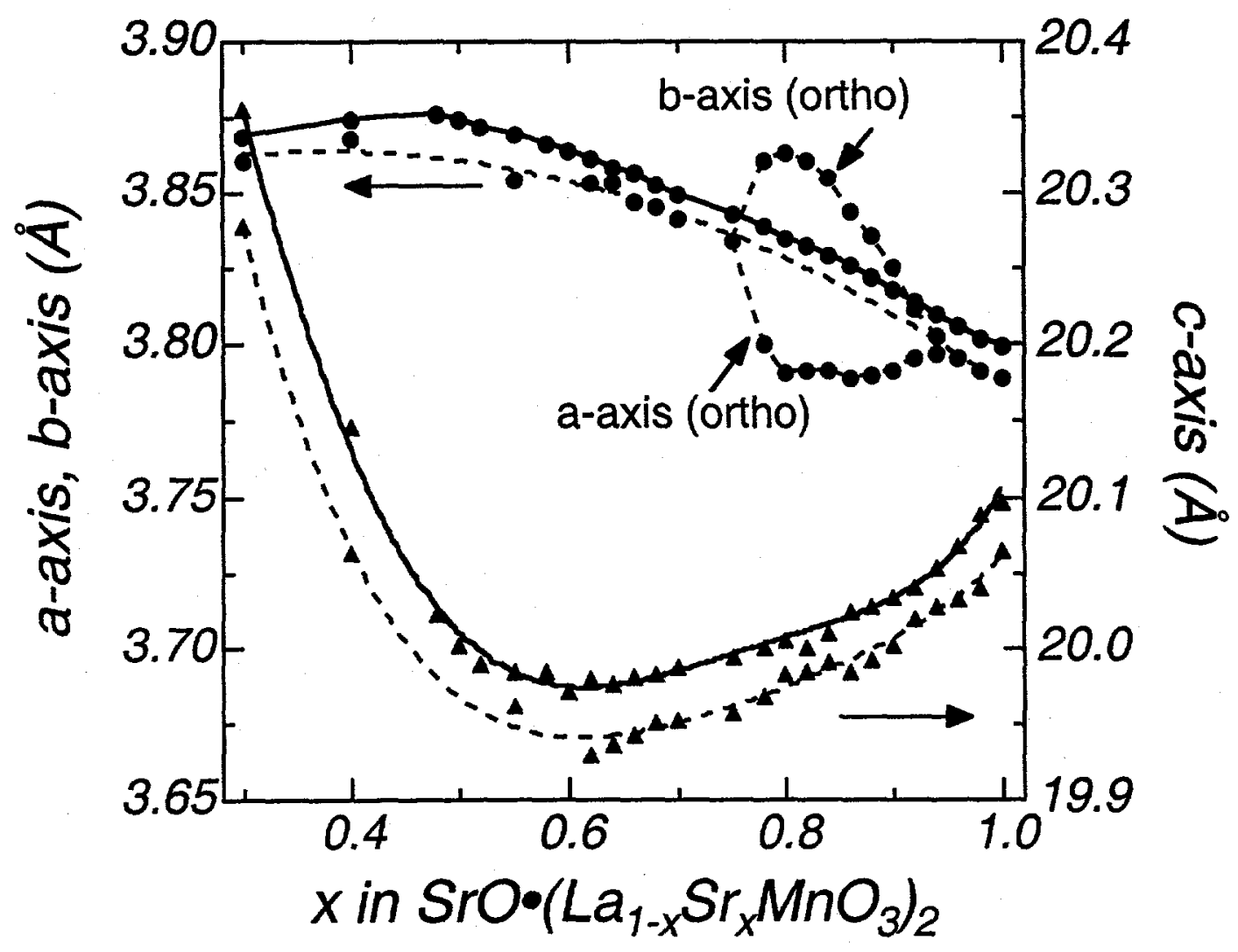

Figure 3 -- Mitchell, et al. "Magnetic Phase Diagram ... " 